\title{
Guest Editors' Introduction: Special Section on the IEEE Pacific Visualization Symposium 2014
}

$\mathrm{T}$ HIS special section of IEEE Transactions on Visualization and Computer Graphics (TVCG) presents extended versions of four selected papers from the 2014 IEEE Pacific Visualization Symposium (PacificVis'14). Past PacificVis symposia were held in Kyoto (2008), Beijing (2009), Taipei (2010), Hong Kong (2011), Songdo (2012), and Sydney (2013). In 2014, PacificVis was back to Japan, and held in Yokohama from March 4th to 7th, 2014.

The successful organization of previous six PacificVis symposia allowed us to introduce a formal two-stage review process for full papers selection. This really helped guarantee the high quality of accepted papers. For fully facilitating this process, we also extended the reviewing period while keeping the fundamental strategies for conducting the review process consistent with the previous years. The selection process was again competitive in the PacificVis'14, and the selected papers were organized into eight full paper sessions: Flow Visualization, Tensor Visualization, Geographic/Geospatial Visualization, Graphs/ Networks, Patterns and Glyphs, Multidimensional Data, Visual Knowledge Discovery, and Data Manipulation and Exploration. Among them, we carefully selected four best papers and invited the respective authors to submit their extended versions to this special section of IEEE TVCG. In what follows, we briefly describe each paper included in this special section.

The paper "Exploring Curved Schematization of Territorial Outlines" presents a new approach toward simulating the composition of hand-drawn schematized maps, by primarily focusing on schematization of circular arcs. The proposed algorithm allows to aesthetically transform curved geographic outlines into schematized forms, where the size and topology of each region is maintained as faithfully as possible. In contrast to conventional schematization techniques for straight lines, this work enriches the expressive power of schematized maps even for low output complexities. The effectiveness of the approach has also been supported by geometric evaluation of the resulting schematized maps and an associated online user study.

In the paper "Semantic Enrichment of Movement Behavior with Foursquare - A Visual Analytics Approach," a new visual analytics approach is proposed for finding meaningful semantics hidden in movement data. This becomes possible by associating such data with detailed geolocated information of points of interest (POIs), in this case using Foursquare as a location-based social media service. For augmenting reliability and trustworthiness of the visualization results, the approach aggressively employs a human-in-the-loop analysis in the decision making phase, by facilitating analysts to interact with various types of spatiotemporal uncertainties arising from inaccuracy in the data. Data with known ground truth has also been introduced to evaluate the applicability of the proposed approach.

The paper "Moment Invariants for 2D Flow Fields via Normalization in Detail" proposes to use moment invariants as pattern descriptors specific to 2D flow fields. This new class of invariants is introduced by generalizing shape descriptors commonly used in the field of image recognition, and formulated as a powerful tool for identifying similar flow structures, even under certain transformations. In particular, the approach employs moment normalization to define a complete and independent set of flow field descriptors that are invariant under translation, rotation, and scaling. This extended version also include detailed theoretical developments together with complete descriptions of the computational algorithms, which are followed by experimental results with a realworld dataset.

The paper "Robustness-Based Simplification of 2D Steady and Unsteady Vector Fields" is also a research work on 2D vector fields, but aimed at simplifying the complexity of the associated flow data. Conventional simplification approaches usually rely on flow topology consisting of critical points and streamlines and thus cannot be sufficiently feasible due to instability in the numerical integration. This work presents a novel simplification approach derived from the recently introduced notion of robustness, which quantifies the stability of critical points with respect to the perturbation of the vector fields. The applicability of the present work is revealed by applying several synthetic and real-world datasets including both static and time-varying cases.

The previous six PacificVis symposia were successfully organized among countries, with the objective to foster greater exchange between visualization researchers and practitioners, and to draw more researchers in the AsiaPacific region to enter this rapidly growing area of research. We have been in a new phase for enhancing the true maturity of the series of the PacificVis symposia themselves, and serving as another opportunity to discuss the state-of-theart techniques in visualization in cooperation with other top-conferences. 
The authors would like to thank Leila de Floriani, the Editor-in-Chief, and Ming Lin, the previous Editor-inChief, for their strong and continued support to us, and the editorial staff of IEEE TVCG for their dedicated efforts on preparing this special section. Our thanks are also extended to anonymous reviewers for their timely and valuable feedback, which greatly helped us to maintain the high-quality of the final papers. We sincerely hope that you will enjoy the extended versions of the top papers presented in PacificVis 2014!!

\author{
Ulrik Brandes \\ Hans Hagen \\ Shigeo Takahashi \\ Xiaoru Yuan \\ Guest Editors
}

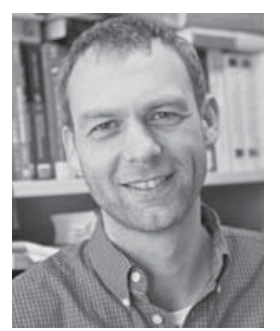

Ulrik Brandes is currently a professor for algorithmics at the University of Konstanz, Germany. $\mathrm{He}$ received the diploma in computer science in 1994 from RWTH Aachen, and the PhD degree in 1999 from the University of Konstanz. Following his habilitation, he became an associated professor at the University of Passau in 2002. His research interests include algorithmics, network science, and information visualization. $\mathrm{He}$ is an area editor of Network Science, an associate editor of Social Networks, and on the editorial board of the Journal of Graph Algorithms and Applications. Since 2007, he has been serving on the board of directors of the International Network of Social Network Analysis.

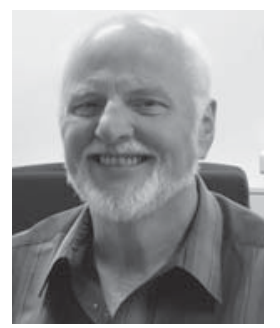

Hans Hagen received the BS degree in computer science 1976 and the MS degree in mathematics from the University of Freiburg 1979, and the $\mathrm{PhD}$ degree in mathematics from the University of Dortmind in 1982. He is currently a professor in the Department of Computer Science, University of Kaiserslautern. His research interests include scientific visualization, visual analytics and geometric modeling. He served as an editor in chief of IEEE TVCG from 1999 to 2002. He received the IEEE Career Award 2009 and the John Gregory Memorial Award 2002.

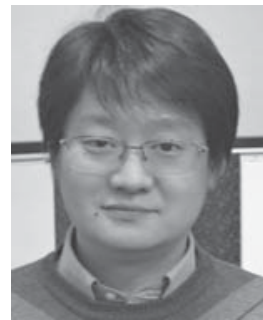

Shigeo Takahashi received the BS, MS, and $\mathrm{PhD}$ degrees in computer science from the University of Tokyo in 1992, 1994, and 1997, respectively. $\mathrm{He}$ is currently a professor in the Department of Computer Science and Engineering, University of Aizu, Japan. His research interests include scientific visualization, geospatial visualization, visual perception modeling, and geometric modeling. He served as an associate editor of IEEE TVCG from 2011 to 2015 , as a program cochair of PacificVis 2014, 2015 and $\mathrm{VINCI}$ 2015 , and as a program committee member for more than 50 visualization and graphics conferences. He received the Most Cited Paper Award for Graphical Models (2004-2006) from Elsevier in 2007.

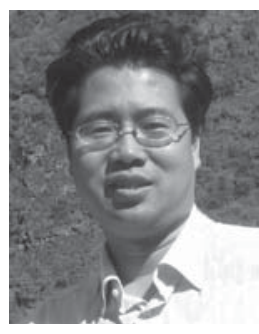

Xiaoru Yuan is a faculty member in the School of Electronics Engineering and Computer Science and the vice director in Information Science Center, Peking University. He received the $\mathrm{PhD}$ degree in computer science in 2006, from the University of Minnesota at Twin Cities. His primary research interests are in the field of scientific visualization, information visualization and visual analytics. He has coauthored more than 60 technical papers in IEEE VIS, EuroVis, IEEE PacificVis, IEEE TVCG, and other major international visualization conference and journals. His coauthored work on high dynamic range volume visualization received Best Application Paper Award at the IEEE Visualization 2005 conference. 